\begin{tabular}{llll}
\hline DE & DE GRUYTER & $\begin{array}{c}\text { HUNGARIAN JOURNAL OF } \\
\text { INDUSTRY AND CHEMISTRY } \\
\text { Vol. 45(1) pp. 29-36 (2017) } \\
\text { hij. mk.uni-pannon.hu } \\
\text { DOl: } 10.1515 / \mathrm{hic}-2017-0006\end{array}$
\end{tabular}

\title{
FORMATION, PHOTOPHYSICS, PHOTOCHEMISTRY AND QUANTUM CHEMISTRY OF THE OUT-OF-PLANE METALLOPORPHYRINS
}

\author{
Zsolt Valicsek, ${ }^{1 *}$ Melitta P. KIss, ${ }^{1}$ Melinda A. Fodor, ${ }^{1}$ Muhammad ImRan, ${ }^{2}$ \\ AND OTTÓ HORVÁTH ${ }^{1}$ \\ ${ }^{1}$ Department of General and Inorganic Chemistry, Institute of Chemistry, Faculty of Engineering, \\ University of Pannonia, Egyetem u. 10., H-8200 Veszprém, HUNGARY \\ ${ }^{2}$ Department of Chemistry, Baghdad-ul-Jadeed Campus, The Islamia University of Bahawalpur, \\ 63100 Bahawalpur, PAKISTAN
}

\begin{abstract}
Among the complexes of porphyrins, special attention has been paid to those possessing out-of-plane (OOP) structures, for the formation of which the size, as well as the coordinative character of the metal center are responsible. In these coordination compounds, the central atom cannot fit coplanarly into the cavity of the ligand, hence, it is located above the porphyrin plane, distorting it. Equilibria and kinetics of the complex formation, spectrophotometric, photophysical and primary photochemical properties of post-transition and lanthanide OOP metalloporphyrins were investigated, in addition electronic structural calculations were performed; hence, the general OOP characteristics were determined.Meanwhile, few doubtful questions have attempted to be answered concerning the categorization of metalloporphyrins, the borderline case complexes and hyperporphyrins.
\end{abstract}

Keywords: out-of-plane metalloporphyrins, formation kinetics, UV-Vis spectrophotometry, photochemistry, borderline case complexes

\section{Introduction}

Porphyrins and their derivatives play important roles in several biochemical systems. Four pyrroles are connected through methylidine bridges, forming the porphin ring. Its planar structure with an extended conjugated $\pi$-electron system provides aromatic characteristics and a special coordination cavity for the binding of metal ions of suitable radius [1-2].

Metalloporphyrins are the central parts of naturally important compounds, e.g., magnesium(II) chlorins in bacteriochlorophylls and chlorophylls; iron(II) protoporphyrin in hemoglobin; and iron(III) protoporphyrins in myoglobin, cytochromes, oxidase, peroxidase, catalase, and oxoanion reductase enzymes. Ringed tetrapyrroles provide strong chelating effect which can promote the hyperaccumulation of rare metal ions in living cells, and also in abiotic environments, e.g. in kerogens.

In porphyrins the conjugation favors a planar structure. However, peripheral substituents or the metal center (originating from its size or axial ligand) can cause geometrical distortion. This certainly affects the functions of enzymes, as well as the biosynthesis of metalloporphyrins. In chemical research, due to distortion, redox potentials, basicity, reactivity, catalytic

*Correspondence: valicsek@almos.uni-pannon.hu activity and coordinative abilities of porphyrins can be modified. Also, due to the distortion, the degree of symmetry decreases, resulting in characteristic spectral changes in various ranges of the electromagnetic spectrum. The most frequent types of distortions are dome, saddle, ruffled and wave (chair-like) [3].

Overcrowded substitution on the periphery [3-4] or insufficiently short metal-nitrogen bonds due to the shrinkage of the coordination cavity can cause the ruffled or saddled deformation [2, 5-7]. If, however, the $\mathrm{M}-\mathrm{N}$ bonds are significantly longer than half the length of the diagonal N-N distance in the free-base porphyrin, dome deformation can occur. This happens if the radius of the metal center exceeds the critical value of about 75-90 pm (depending on the type of porphyrin ligand) or square planar coordination is not preferred. Such metal ions are too big to fit into the ligand cavity. Hence they are located above the plane of the pyrrolic nitrogens; forming sitting-atop (SAT) or out-of-plane (OOP - see Fig.1) complexes, displaying thermodynamic instability, kinetic lability, typical photophysical features and photochemical reactivity [89].

In this work, we review our recent results regarding the formation, structure and photoinduced behavior of water-soluble OOP metalloporphyrins. These complexes with a diverse range of metal ions can be more simply produced in aqueous systems than in organic solvents. In this regard one of the most suitable free-base ligands is the anionic 5,10,15,20-tetrakis(4sulfonatophenyl)porphyrin $\left(\mathrm{H}_{2} \mathrm{TSPP}^{4-}\right.$ - see Fig. 1) due 
to its negative charge promoting the coordination of positively charged metal ions. Besides, this ligand is the most frequently used reagent among the free-base porphyrins [1].

\section{Experimental}

Analytical grade tetrasodium 5,10,15,20-tetrakis(4sulfonatophenyl)porphyrin $\left(\mathrm{C}_{44} \mathrm{H}_{26} \mathrm{~N}_{4} \mathrm{O}_{12} \mathrm{~S}_{4} \mathrm{Na}_{4} \cdot 12 \mathrm{H}_{2} \mathrm{O}\right.$ $=\mathrm{Na}_{4} \mathrm{H}_{2}$ TSPP $\cdot 12 \mathrm{H}_{2} \mathrm{O}$ ) (Sigma-Aldrich) and simple metal salts such as nitrate, sulfate, chloride or perchlorate were used for the experiments. The solvent was double-distilled water purified with a Millipore Milli-Q system. The $\mathrm{pH}$ of the majority of the metalloporphyrin solutions was adjusted to 8 by application of a borate buffer, whilst maintaining the ionic strength at a constant value of $0.01 \mathrm{M}$. In a few cases, the $\mathrm{pH}$ was regulated to 6 , and the ionic strength to $1 \mathrm{M}$, by an acetate buffer, to hinder hydrolysis.

The absorption spectra were recorded and the spectrophotometric titrations were monitored by using a Specord S-100 or a Specord S-600 diode array spectrophotometer. For the measurement of fluorescence spectra, a Perkin Elmer LS-50B or a Horiba Jobin Yvon FluoroMax-4 spectrofluorometer was applied. The latter piece of equipment supplemented with a time-correlated single photon counting (TCSPC) accessory was utilized to determine fluorescence lifetimes, too. UV-Vis spectrophotometric data (molar absorption, fluorescence quantum yields and lifetimes) of the free-base porphyrin were used as references for the determination of those of metalloporphyrin complexes [1].

For the determination of photochemical properties via continuous irradiations, a piece of AMKO LTI photolysis equipment (containing a 200W Xe-Hg lamp and a monochromator) was applied.

For the electronic structural calculations, the B3LYP Density Functional Theory (DFT) method and the LANL2DZ basis set were used. On the basis of our earlier quantum chemistry experiences, the sulfonatophenyl substituents exhibit negligible effects on the coordination of the metal center in the cavity; thus, the anionic porphyrin $\left(\mathrm{H}_{2} \mathrm{TSPP}^{4-}\right)$ can be modeled on the unsubstituted porphin $\left(\mathrm{H}_{2} \mathrm{P}\right)[4,10]$.

\section{Results and discussion}

\subsection{UV-Vis spectrophotometry}

Porphyrins and their derivatives belong to the strongest light-absorbing materials (both natural and artificial), therefore, ultraviolet-visible spectrophotometry is one of the most fundamental, in addition, most informative spectroscopic techniques in porphyrin chemistry. They possess two $\pi \pi^{*}$ electronic transitions in the visible range of the electromagnetic spectrum: B- or Soret band at about 350-500 nm, usually with a molar absorbance of $10^{5} \mathrm{M}^{-1} \mathrm{~cm}^{-1}$ (Fig.2), and Q bands at 500-750 nm
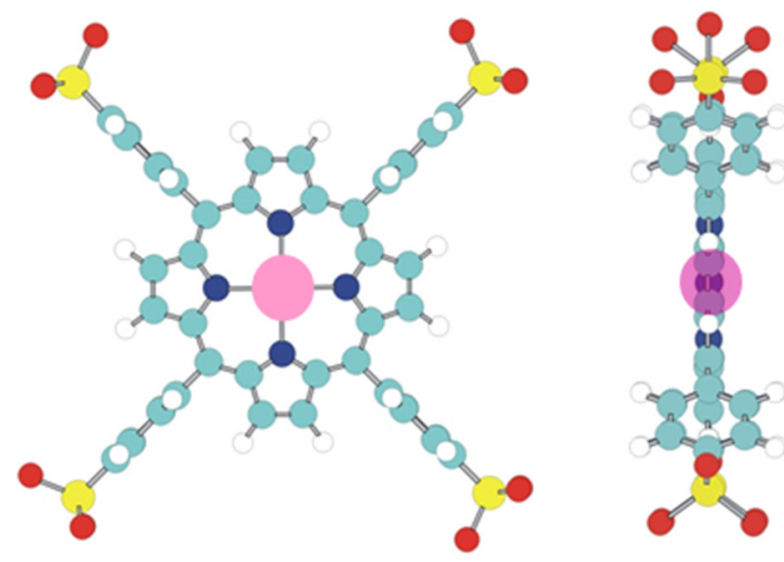

a)
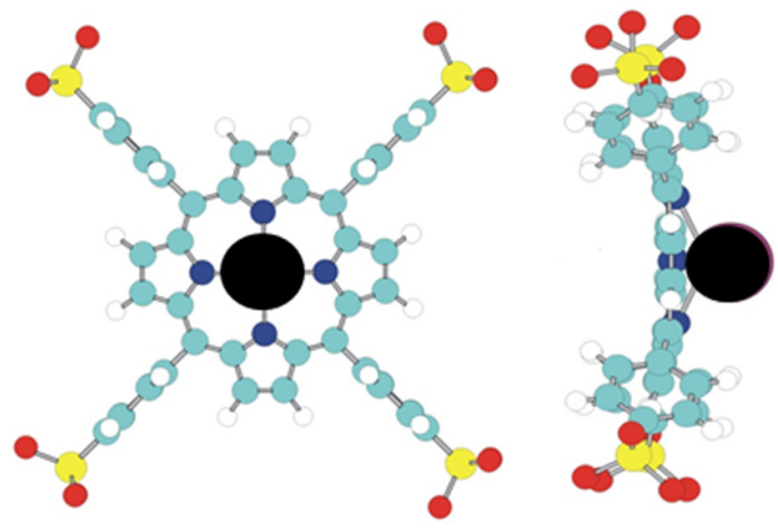

b)

Figure 1. Structure of an in-plane metalloporphyrin $\{$ MTSPP $=$ metallo-5,10,15,20-tetrakis $(4-$

sulfonatophenyl)porphyrin $\}$ (a);

and that of an out-of-plane complex (b) [9].

generally with intensities of one order of magnitude less. These latter bands in free-base ligands split due to the presence of protons on two diagonally situated pyrrolic nitrogens, to be more precise, as a result of the reduced symmetry (because of the disappearance of the four-fold rotation axis) compared to the metallated or deprotonated forms. This split is not detectable in the Soret range, hence, these two types of bands in the visible region are remarkably different [1].

In the Soret region, compared to the corresponding free-base ligands, the typical in-plane metalloporphyrins (e.g. $\mathrm{Fe}^{3+}, \mathrm{Au}^{3+}, \mathrm{Cu}^{2+}, \mathrm{Pd}^{2+}$ ) exhibit blueshifts because the atomic orbitals of their metal centers which are covalently bonded in the plane can overlap more strongly with the highest occupied molecular orbitals (HOMO) of the ligand, resulting in a stronger reduction in energy; whereas the lowest unoccupied molecular orbitals (LUMO) do not change. Thus, the energy gaps between the excited and ground states become greater. In the OOP complexes, the atomic orbitals of the more weakly bonded metal ions (e.g. $\mathrm{Cd}^{2+}, \mathrm{Hg}^{2+}, \mathrm{Tl}^{3+}$ ) may slightly affect the unoccupied MOs and to a lesser extent the occupied ones, leading to a reduction of the energy gaps, i.e. an increase in the corresponding wavelengths (Scheme 1) [1,9]. 


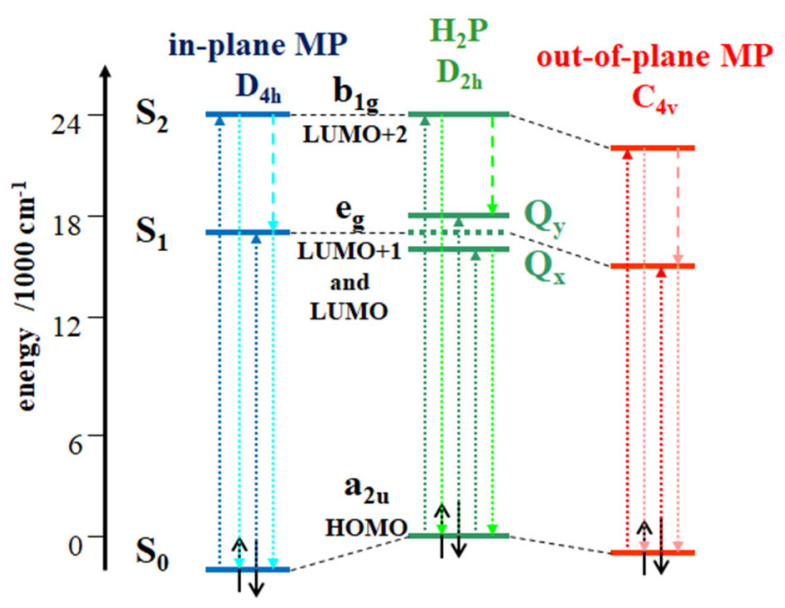

Scheme 1. Simplified energy level diagram for the change of the porphyrin's molecular orbitals in different types of complexes [9].

Beside electronic factors, due to the rigidity of the porphyrins' ringed structure, steric effects also influence the spectra: the redshift of absorption bands is one of the most common spectroscopic consequences of the non-planarity of porphyrin [3]. Octabrominated freebase porphyrin, $\mathrm{H}_{2} \mathrm{TSPPBr}_{8}{ }^{4-}$, was applied to investigate the spectrophotometric effects of the macrocycle's highly saddle-distorted structure (Fig.2). In porphyrins with aryl substituents, this distortion can lead to the extension of delocalization by the twisting of aryl substituents from a nearly perpendicular orientation closer to the porphyrin plane (Fig. 1) [4].

The larger, post-transition metal ions, e.g. thallium(I), lead(II) and bismuth(III) ions, can cause a similarly large redshift of the porphyrins' absorption bands. Since their complexes possess the most highly dome-distorted structures, also a ruffled-like deformation of the periphery superposes on this high degree of doming. Considering the spectral effects (bathochromic or not quite exactly hyperchromic effects), the complexes possessing such highly redshifted absorption bands used to be referred to as hyperporphyrins; depending on the highest occupied electron subshell of the metal center, p- or d-type hyperporphyrins. Previously in terms of this categorization of metalloporphyrins, only the electronic effects of the metal ion (through its electron configuration) were taken into consideration and not steric (distorting) effects [1]. Nevertheless, in the typical d-type hyperporphyrins, e.g. the low-spin chromium(III), manganese(III), nickel(II) and cobalt(III) porphyrins, the radius of the metal center, and thus, the metal-nitrogen bonds are too short, resulting in the contraction of the coordination cavity, along with the ruffled deformation of the macrocycle [2, 5-7].

\subsection{Equilibrium and kinetics of complex formation}

Porphyrins are peculiar ligands in terms of complexation due to their planar, cyclic, rigid, aromatic,

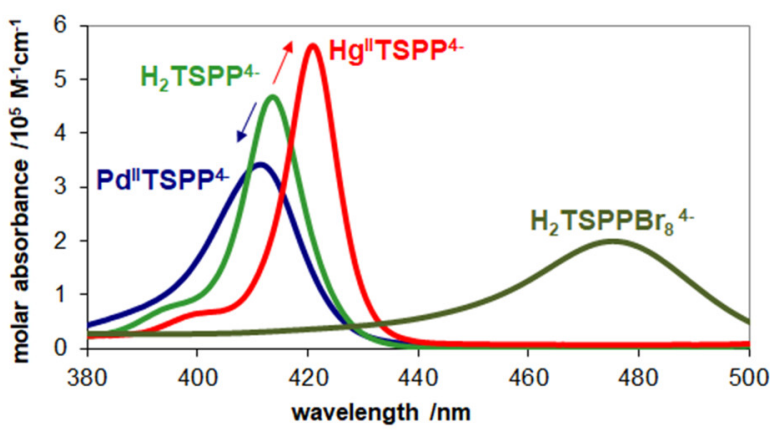

Figure 2. Absorption spectra of the free base $\left(\mathrm{H}_{2} \mathrm{TSPP}^{4-}\right)$; the highly distorted, octabrominated free base $\left(\mathrm{H}_{2} \mathrm{TSPPBr}_{8}{ }^{4}\right)$; a typical in-plane $\left(\mathrm{Pd}^{\mathrm{II}} \mathrm{TSPP}^{4-}\right)$; and a typical out-of-plane metalloporphyrin $\left(\mathrm{Hg}^{\mathrm{II}} \mathrm{TSPP}^{4-}\right)$ within the Soret range [1].

tetradentate, as well as protonated structure. The formation of an out-of-plane complex of a large metal ion is usually at least two orders of magnitude faster than that of an in-plane one since a smaller metal ion is not able to coordinate to all four pyrrolic nitrogens of the reaction intermediate, in the cavity of which the two protons also remain $\left\{\mathrm{H}_{2}-\mathrm{P}-\mathrm{M}\right\}$. Therefore, dissociation of the metal ion is more favorable than that of the protons. Besides the insertion of a smaller metal ion, its dissociation from the in-plane complex of the endproduct may be kinetically hindered due to the rigidity of the macrocycle [1].

Formation of the in-plane complexes used to be enhanced by the addition of a small concentration of a metal ion with larger ionic radius (e.g. $\mathrm{Cd}^{2+}, \mathrm{Hg}^{2+}, \mathrm{Pb}^{2+}$ ) to the solution of the smaller one because the insertion of the larger metal ion into the ligand cavity is much faster. However, the OOP complex is considerably less stable. In its dome-distorted structure, two diagonal pyrrolic nitrogens are more accessible from the other side of the ligand, owing to the enhancement of their $\mathrm{sp}^{3}$ hybridization, hence, the metal center can be easily exchanged for the smaller one [1-2, 9].

This accessibility makes the realization of dinuclear out-of-plane monoporphyrins (2:1 complexes) possible if the metal ion possesses a low (single) positive charge and is large, i.e. its charge density is small enough, e.g. mercury(I), silver(I) and thallium(I) ions [1, 8-9].

Moreover, the out-of-plane position of the metal center, together with the dome-distorted structure (owing to the twisting of aromatic substituents from a nearly perpendicular position closer to the porphyrin plane) may promote the formation of so-called sandwich complexes of various compositions, in which two metal ions can coordinate to one macrocycle, and, reversely, one metal ion can concomitantly coordinate to two ligands, (Fig.3) [1-2, 9]. Lanthanide(III) ions form typical examples of metallo-oligoporphyrins because they are inclined to form complexes of higher coordination number (8-12). However, they are hard Lewis acids, hence, their insertion into the coordination cavity of the softer $\mathrm{N}$-donor porphyrin ligand is a slow and complicated process in aqueous solutions. This 
phenomenon partly originates from the stability of their aqua complexes. Due to the consequence of their Pearson-type hard character, they coordinate rather to the peripheral substituents of porphyrin (instead of the pyrrolic nitrogens), i.e. to the ionic group ensuring water-solubility if they possess similarly hard O-donor atoms (e.g. carboxy or sulfonatophenyl groups). At lower temperatures, under kinetic control, the early lanthanide(III) ions are not able to coordinate into the cavity, rather to the periphery; resulting in the formation of the tail-to-tail dimer of free-base ligands (as the tail used to be referred to as the periphery). Higher temperatures and thermodynamic control are also necessary for the insertion of metal ions into the cavity produced by four pyrrolic nitrogens; resulting in the formation of typical metalloporphyrin complexes. After the discovery of the possible coordination bonds between lanthanide ions and sulfonato substituents, the formation of lanthanide bisporphyrins may be realized as a tail-to-tail dimerization of two metallomonoporphyrin complexes through a metal bridge; deviating from the head-to-head connection as in the case of typical sandwich complexes (head refers to the cavity; see Fig.3). On the basis of our previous experiences, the coordination position of lanthanide ions was influenced by the change in temperature [1-2], [11$13]$.

During the investigation of the formation of "typical p-type hyperporphyrin" complexes (e.g. $\mathrm{Tl}^{+}$, $\left.\mathrm{Pb}^{2+}, \mathrm{Bi}^{3+}\right)$, the species possessing highly redshifted absorption bands are the end-products of metalation only in hydrophobic solvents, since they can appear in aqueous solutions as intermediates with shorter or longer lifetimes depending on the metal ion. The absorption spectra of the end-products of these transformation reactions are very similar (less redshifted) to those of the typical, common out-of-plane metallo-monoporphyrins (e.g. $\mathrm{Hg}^{\mathrm{II}}$-porphyrin in Fig.2). This phenomenon may be accounted for to the considerable coordination ability or the polarizing effect of water molecules, which can enable the complex to overcome the kinetic energy barrier towards the formation of the more stable structure, in which the metal center is located closer to the ligand plane, resulting in decreases in distortion, as well as redshift. Furthermore, "hyperporphyrins" can appear as intermediates in smaller amounts during the formation of typical, common out-of-plane metallomonoporphyrins as well $[1,14]$.

In the case of "d-type hyperporphyrin" complexes (e.g. $\mathrm{Mn}^{3+}, \mathrm{Co}^{3+}, \mathrm{Ni}^{2+}$ ), the low-spin and ruffled complex with a contracted cavity can exist in a spinisomerization equilibrium with the high-spin and planar forms, which not only exhibits less redshift, but rather blueshifted absorption bands compared to those of the free-base ligand. This reaction can be influenced by the strength of the M-N bonds (owing to the electronic effects of peripheral or axial substituents), due to the size of the coordination cavity (owing to the substitution or saturation of methylidene bridges or pyrrolic carbons) $[1-2,5-7]$.

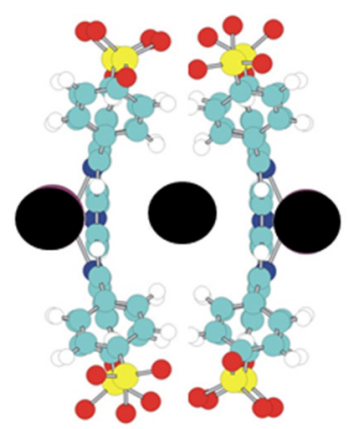

a)

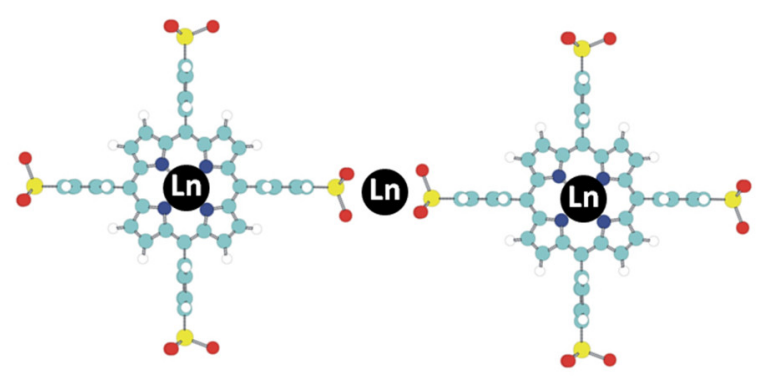

b)

Figure 3. Potential structures of $3: 2$ bisporphyrin:

(a) head-to-head or (b) tail-to-tail [2].

\subsection{Photophysics}

Porphyrins represent one of the most interesting groups of compounds in terms of photophysical properties and biological significance. Due to their rigid structure and aromatic electronic system, they display two types of fluorescence: beside their relatively strong singlet-1 fluorescence in the range of 550-800 nm, weak and rare singlet-2 luminescence is observable between 400 and $550 \mathrm{~nm}$ upon excitation of the Soret band (Scheme 1) [1].

The quantum yields of $\mathrm{S}_{2}$-fluorescence are about 3 orders of magnitude lower than those of $\mathrm{S}_{1}$-fluorescence in the case of free-base porphyrins, especially $\sim 1200$ fold for $\mathrm{H}_{2} \mathrm{TSPP}^{4-}\left(6.3 \times 10^{-5}\right.$ vs. $\left.7.5 \%\right)$. However, this ratio decreases with metalation, mainly in the case of the formation of out-of-plane complexes. Since the structure of $\mathrm{S}_{2}$-excited porphyrins may be close to that of the dome-distorted OOP complexes that are already in the electronic ground state. Another consequence of this structural similarity (namely small Stokes shift) is that the directions of the shifts of $\mathrm{S}_{2}$-fluorescence bands invert (according to Soret absorption) between the inplane (redshifted) and out-of-plane (blueshifted) complexes when compared to the free-base ligand [1].

Singlet-1 fluorescence bands exhibit blueshifts in both types (in-plane and out-of-plane) of metalloporphyrins, as a consequence of the aforementioned split in free-base ligands because of the presence of two protons, as well as their reduced symmetry (Scheme 1). Furthermore, almost all complexes exhibit similarly large Stokes shifts, as well as lifetimes and quantum yields. In the case of in-plane 
metal centers, the spin-orbit coupling, as an electronic quenching effect, may be dominant. Whereas for typical out-of-plane metalloporphyrins, the distortion, as a steric effect, can enhance their non-radiative decay.

The highly distorted (d- and p-type) hyperporphyrins, the paramagnetic in-plane complexes (e.g. $\mathrm{Fe}^{\mathrm{III}} \mathrm{TSPP}^{3-}$ ), as well as the head-to-head-type OOP bisporphyrins \{e.g. $\left.\mathrm{Hg}_{3}{ }_{3}(\mathrm{TSPP})_{2}{ }^{6-}\right\}$ do not exhibit significant levels of luminescence at room temperature. Conversely, the paramagnetic out-of-plane complexes (e.g. $\quad \mathrm{Ln}^{\mathrm{III}} \mathrm{TSPP}^{3-}$ ) possess similar fluorescence properties to that of the diamagnetic ones because a paramagnetic metal ion can cause the disappearance of fluorescence by spin-orbit coupling only if it is located in the plane. In the OOP position, it is not able to perturb as efficiently the molecule orbitals of the macrocycle that result in the common absorption and emission out-of-plane characteristics [1-2,9].

Lanthanide(III) bisporphyrins $\left\{\operatorname{Ln}_{3}^{\mathrm{III}}{ }_{3}(\mathrm{TSPP})_{2}{ }^{3-}\right\}$ have many similarities in terms of absorption and emission properties to those of monoporphyrin complexes $\left(\mathrm{Ln}^{\mathrm{III}} \mathrm{TSPP}^{3-}\right)$. These may only originate from the very weak $\pi-\pi$ interactions between the macrocycles in the tail-to-tail-type aggregations (Fig.3) [1-2, 11-13].

\subsection{Photochemistry}

Porphyrin derivatives are the main components of photosynthesis, synthetically as well. Since the overall quantum yield of fluorescence and intersystem crossing resulting in the formation of triplet states is in excess of $95 \%$, merely a slight proportion of excitation energy is dissipated as heat from singlet states. This ratio is the major reason why porphyrins are efficient in terms of optical sensations and photosensitizations. Free-base and kinetically inert in-plane metalloporphyrins may be appropriate candidates to be applied in photocatalytic systems based on outer-sphere electron transfer. D-type hyperporphyrins can be particularly promising from this viewpoint owing to their distorted structure which may enhance the (photo)redox reactivity of these coordination compounds. In the presence of a suitable electron acceptor (methylviologen, $\mathrm{MV}^{2+}$ ) and donor (e.g. triethanolamine, TEOA), these complexes proved to be efficaciousl photocatalysts that transfer electrons between the ground-state reactants through an outersphere mechanism, generating the $\mathrm{MV}^{\circ+}$ radical cation. This system can be applied for the production of hydrogen from water [2, 5-7].

Contrarily, the inner-sphere photoredox reactions are characteristic of the out-of-plane metalloporphyrins because of this special coordination (Scheme 2): an irreversible photoinduced charge-transfer from the ligand to the metal center (ligand-to-metal charge transfer, LMCT) improves the efficiency of charge separation, which allows their utilization as catalysts in cyclic processes for the synthesis of chemicals capable of conserving light energy, hopefully in terms of the photochemical cleavage of water. Due to photoinduced LMCT the charge of the metal center decreases and its size increases, overall its charge density diminishes,

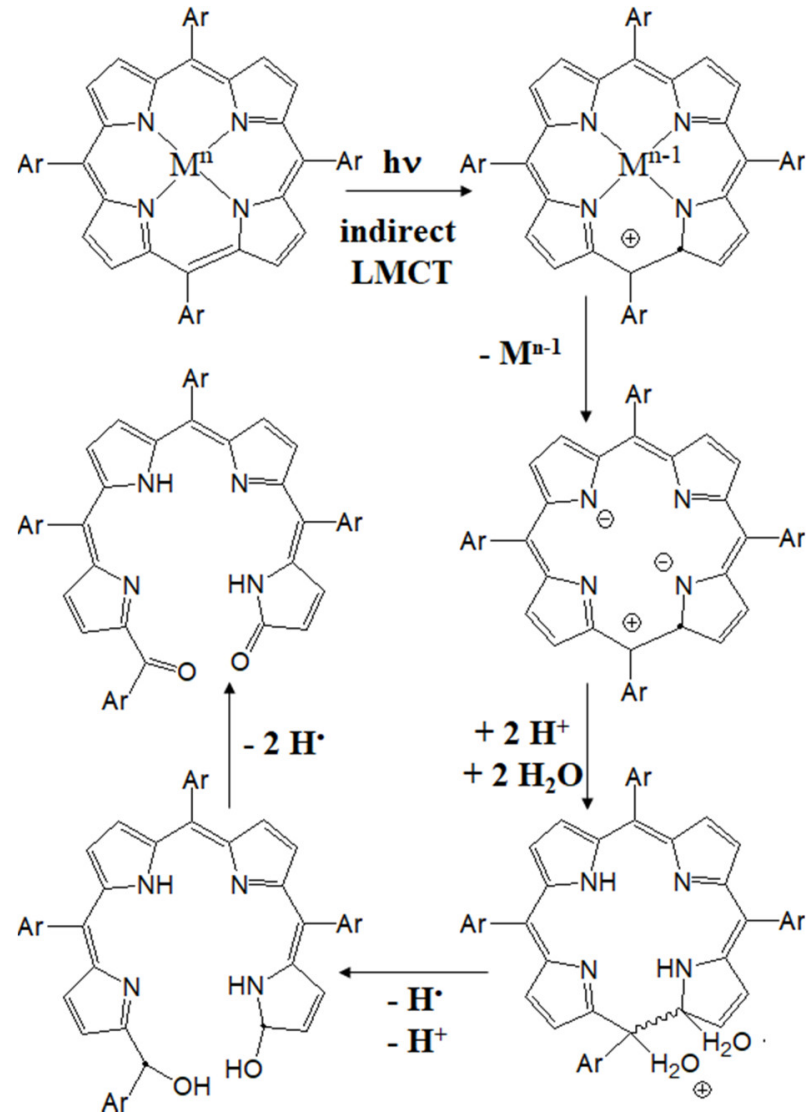

Scheme 2. Simplified demonstration of the mechanism for the inner-sphere photoredox reaction of an out-ofplane metalloporphyrin [8].

hence, the coordinative bonds can easily split. The reduced metal ion can leave the cavity, primarily in polar solvents, and induce further redox reactions. The latter processes strongly depend on the stability of the reduced metalion in the actual medium. The oxidized and metal-free (cat)ionic radical of porphyrin is a very strong base: it is immediately protonated and forms the free-base radical, which is a long-lived and rather strong electron acceptor, especally in deaerated solutions. Since it would only oxidize water to oxygen at higher pHs, a slightly more efficent reducer (such as alcohols or aldehydes of low molecular weight) is needed, from which useful byproducts can be produced in terms of photocatalytic hydrogen generation. In the absence of any electron donor that promotes the regeneration of the porphyrin, it undergoes the primary photochemical processes; an overall four-electron oxidation involving a ring-cleavage, the end-product of which is a dioxotetrapyrrole derivative (bilindione). This ring-opening process can be followed by spectrophotometry owing to the disappearance of the Soret band, as well as the typical change in the region of $Q$ bands [2, 4, 8-16].

Photochemical quantum yields of this ring-opening reaction (without regeneration) are about 2-3 orders of magnitude higher for the out-of-plane complexes $\left(10^{-4}-10^{-2}\right)$ than for the free-base and in-plane metalloporphyrins $\left(10^{-6}-10^{-5}\right)$. In addition, in the case of out-of-plane complexes, photoinduced dissociation in 
the absence of a redox reaction can occur, originating from their lability, and structural transformations to another complex form or conformer were observed in some cases as a photoinduced change of the type or measure of distortion (e.g. d- and p-type hyperporphyrins) [2, 4, 8-10, 14-16].

Besides the typical post-transition metal ions, lanthanide(III) ions were also applied for out-of-plane coordination because their contraction makes the finetuning of the out-of-plane distance possible, and their high negative redox potentials promote the photoinduced cleavage of water. Photochemical activities of their complexes confirm that the redox potentials of the metal centers are not the main determining factor, rather their out-of-plane distances [2, 11-13].

Deviating from the OOP complexes of posttransition metal ions, another stable photoproduct was observable during the photolysis of lanthanide(III) porphyrins. It displays a typical absorption band in the $\mathrm{Q}$ range (at $\sim 600 \mathrm{~nm}$ ), which may be assigned as a charge transfer between the metal ion and open-chain, dioxo-tetrapyrrole derivative (bilindione, see Scheme 2). Its oxo-groups, as donor atoms, may coordinate with the lanthanide ions, as a consequence of their similar Pearson-type hard characteristics, contrary to the softer post-transition metal ions $[2,11]$.

During the photolysis experiments, only small differences appeared between lanthanide(III) mono- and bisporphyrin complexes, which might confirm a special type of aggregation through the peripheral sulfonato substituents with weak $\pi-\pi$ interactions (tail-to-tail, see Fig.3) [2, 11-13]. Deviating from these observations, the differences are much more significant in the case of the most typical, post-transition metallo-bisporphyrin compared to the monoporphyrin equivalent; namely between $\mathrm{Hg}_{3}{ }_{3}^{\mathrm{II}}(\mathrm{TSPP})_{2}{ }^{6-}$ and $\mathrm{Hg}^{\mathrm{II}} \mathrm{TSPP}^{4-}$. The overall quantum yield is $\sim 2$ orders of magnitude higher and the photoinduced dissociation of a metal ion became the dominant reaction in the head-to-head sandwich complex as a consequence of the strong $\pi-\pi$ interactions [9-10].

\subsection{Quantum chemical calculations}

The main aims of our electronic structural calculations were to determine the primary consequences for the outof-plane position of a metal center, and confirm the experimentally observed correlation between the UVVis spectral shifts and the coordination position of the metal center (in-plane or out-of-plane). In the light of these aspects, the unsubstituted porphin $\left(\mathrm{H}_{2} \mathrm{P}, \mathrm{C}_{20} \mathrm{H}_{14} \mathrm{~N}_{4}\right)$ was used as a model for the calculations, instead of the tetrakis(sulfonatophenyl)porphyrin $\quad\left(\mathrm{H}_{2} \mathrm{TSPP}^{4-}\right.$, $\mathrm{C}_{44} \mathrm{H}_{26} \mathrm{~N}_{4} \mathrm{O}_{12} \mathrm{~S}_{4}{ }^{4-}$ ). On the basis of the few comparative calculations that were conducted, the phenyl-, as well as the sulfonatophenyl substituents have negligible effects on the coordination of the metal center in the cavity. However, they can significantly influence the formation of bisporphyrin complexes, even in the case of head-tohead structures $[4,10]$.
According to our quantum chemical experience, the value of the critical radius became $\sim 100$ pm instead of the experimentally suggested $\sim 75-90 \mathrm{pm}$ as a consequence of the significant expansion of the coordination cavity to coplanarly incorporate the metal ions. The proportion of borderline cases, i.e. complexes with questionable structures (somewhere between inplane and out-of-plane), increased with further posttransition metal ions (e.g. $\mathrm{Ag}^{2+}[15], \mathrm{Cd}^{2+}[4], \mathrm{Tl}^{3+}[16]$ ) that possess ionic radii of $\sim 90-95 \mathrm{pm}$. Calculated bond lengths $(\mathrm{M}-\mathrm{N})$ and atomic distances $(\mathrm{N}-\mathrm{N})$ considerably deviate from the expected ones supposed on the basis of the values of the deprotonated porphyrins $\left(\mathrm{P}^{2-}\right)$. To describe this phenomenon, an axial ligand was applied to these metal centers to extract them out of the cavity. Consequently, expansion stopped, and the out-of-plane distance increased dramatically together with the degree of dome distortion and redshifts of absorption bands. From this point of view, two possible explanations can be supposed for the borderline-case complexes: the experimentally observed common OOP characteristics may originate from this expansion, tension; and small perturbations (e.g. the axial coordination in the calculation or photoexcitation in the experiments) may facilitate the metal center to adopt an out-of-plane position, too. Another possibility is that the method of calculation strongly prefers planar structures.

In our time-dependent density functional theory (TD-DFT) calculations, the correlation found between the measured and calculated shifts associated with the position of the metal center was not totally linear, but nevertheless acceptable. The main exceptions were the borderline cases (high-spin $\mathrm{Mn}^{2+}, \mathrm{Fe}^{2+}$ and $\mathrm{Zn}^{2+}$ ) and the d-type hyperporphyrins (because their structures were determined to be totally planar), as well as the p-type hyperporphyrins (because a ruffled-like deformation did not superpose on their dome-like structure).

The regression of correlation was much worse within the Soret band than in the case of the Q bands. The Soret band was also split in the calculations, which cannot be detected experimentally. On the basis of further experimental observations and doubts in the literature, the validity of the theoretical model in use at present is questionable. Hence, the development of a more suitable one is in progress.

\section{Conclusion}

In conclusion, it can be declared that the categorization of metalloporphyrins was complemented by the role of their distortion, which is primarily responsible for their spectral features, whereas the electronic structure of their metal centers is a secondary factor, with a considerable level of emphasis on the in-plane complexes. The position of the metal center (in-plane or out-of-plane) in the monoporphyrin complexes, as well as the type (head-to-head or tail-to-tail) of the bisporphyrin complexes can be determined on the basis of their UV-Vis absorption and emission properties. 
Hyperporphyrin spectra can appear, owing to the peripheral substitution (octabromination) of free-base ligands. Furthermore, the high degree of redshift may disappear during the spin isomerization of d-type metalloporphyrins or the transformation of $p$-type ones. Consequently, the real origin cannot be an electronic but rather a steric effect, namely the measure of distortion, which can confirm the absence of their fluorescence.

In terms of photochemical activity, several dissimilarities were found between the in-plane and outof-plane metalloporphyrins; the most remarkable of them was the mechanism of their photoredox reactions: outer-sphere electron transfer is typical of the previous ones, while the inner-sphere equivalent is most prevalent for the latter ones. As a further consequence of the OOP position of the metal center, photoinduced dissociation and transformation reactions can occur within their complexes.

In our electronic structural calculations, the number of borderline-case complexes expanded, on the basis of which common OOP characteristics that can be experimentally observed may acquire a novel explanation.

\section{Acknowledgement}

This research was supported by the Széchenyi 2020 Fund under the GINOP-2.3.2-15-2016-00016 and EFOP-3.6.1-16-2016-00015 projects.

Assistance with quantum chemical calculations provided by Professor György Lendvay (Research Centre for Natural Sciences, Hungarian Academy of Sciences) is gratefully acknowledged.

Finally, this manuscript is dedicated to the memory of Professor János Liszi, who, as the head of the Doctoral School for Chemistry at the University of Veszprém, praised the corresponding author's PhD dissertation of a similar title in 2007.

\section{REFERENCES}

[1] Valicsek, Z.; Horváth, O.: Application of the electronic spectra of porphyrins for analytical purposes: the effects of metal ions and structural distortions, Microchem. J., 2013 107, 47-62 DOI: 10.1016/j.microc.2012.07.002

[2] Horváth, O.; Valicsek, Z.; Fodor, M.A.; Major, M.M.; Imran, M.; Grampp, G.; Wankmüller, A.: Visible light-driven photophysics and photochemistry of water-soluble metalloporphyrins, Coord. Chem. Rev., 2016 325, 59-66 DOI: 10.1016/j.ccr.2015.12.011

[3] Shelnutt, J.A.; Song, X.-Z.; Ma, J.-G.; Jia, S.-L.; Jentzen, W.; Medforth, C.J.: Nonplanar porphyrins and their significance in proteins, Chem. Soc. Rev., 1998 27, 31-41 DOI: 10.1039/A827031Z
[4] Valicsek, Z.; Horváth, O.; Lendvay, G.; Kikaš, I.; Škorić, I.: Formation, photophysics, and photochemistry of cadmium(II) complexes with 5,10,15,20-tetrakis(4-sulfonatophenyl)porphyrin and its octabromo derivative: the effects of bromination and the axial hydroxo ligand, J. Photochem. Photobiol. A, 2011 218, 143-155 DOI: 10.1016/j.jphotochem.2010.12.014

[5] Fodor, M.A.; Horváth, O.; Fodor, L.; Grampp, G.; Wankmüller, A.: Photophysical and photocatalytic behavior of cobalt(III) 5,10,15,20-tetrakis(1methylpyridinium-4-yl)porphyrin, Inorg. Chem. Commun., 2014 50, 110-112 DOI: 10.1016/j.inoche.2014.10.029

[6] Fodor, M.A.; Horváth, O.; Fodor, L.; Vazdar, K.; Grampp, G.; Wankmüller, A.: Photophysical and photochemical properties of manganese complexes with cationic porphyrin ligands: Effects of alkyl substituents and micellar environment, J. Photochem. Photobiol. A, 2016 328, 233-239 DOI: 10.1016/j.jphotochem.2016.06.011

[7] Major, M.M.; Horváth, O.; Fodor, M.A.; Fodor, L.; Valicsek, Z.; Grampp, G.; Wankmüller, A.: Photophysical and photocatalytic behavior of nickel(II) 5,10,15,20-tetrakis(1-methylpyridinium-4-yl) porphyrin, Inorg. Chem. Commun., 2016 73, 1-3 DOI: 10.1016/j.inoche.2016.09.001

[8] Horváth, O.; Valicsek, Z.; Harrach, G.; Lendvay, G.; Fodor, M.A.: Spectroscopic and photochemical properties of water-soluble metalloporphyrins of distorted structure, Coord. Chem. Rev., 2012 256, 1531-1545 DOI: 10.1016/j.ccr.2012.02.011

[9] Horváth, O.; Huszánk, R.; Valicsek, Z.; Lendvay, G.: Photophysics and photochemistry of kinetically labile, water-soluble porphyrin complexes, Coord. Chem. Rev., 2006 250, 1792-1803 DOI: 10.1016/j.ccr.2006.02.014

[10]Valicsek, Z.; Lendvay, G.; Horváth, O.: Equilibrium, photophysical, photochemical and quantum chemical examination of anionic mercury(II) mono- and bisporphyrins, J. Phys. Chem. B, 2008 112(46), 14509-14524 DOI: 10.1021/jp804039s

[11]Imran, M.; Szentgyörgyi, C.; Eller, G.; Valicsek, Z.; Horváth, O.: Peculiar photoinduced properties of water-soluble, early lanthanide(III) porphyrins, Inorg. Chem. Commun., 2015 52, 60-63 DOI: 10.1016/j.inoche.2014.12.016

[12]Kiss, M.P.; Imran, M.; Szentgyörgyi, C.; Valicsek, Z.; Horváth, O.: Peculiarities of the reactions between early lanthanide(III) ions and an anionic porphyrin, Inorg. Chem. Commun., 2014 48, 22-25 DOI: 10.1016/j.inoche.2014.08.001

[13] Valicsek, Z.; Eller, G.; Horváth, O.: Equilibrium, photophysical and photochemical examination of anionic lanthanum(III) mono- and bisporphyrins: the effects of the out-of-plane structure, Dalton Trans., 2012 41, 13120-13131 DOI: 10.1039/C2DT31189E

[14] Valicsek, Z.; Horváth, O.; Patonay, K.: Formation, photophysical and photochemical properties of water-soluble bismuth(III) porphyrins: the role of the charge and structure, J. Photochem. Photobiol. A, 2011 226, 23- 35 DOI: 10.1016/j.jphotochem.2011.10.011 
[15]Harrach, G.; Valicsek, Z.; Horváth, O.: Watersoluble silver(II) and gold(III) porphyrins: the effect of structural distortion on the photophysical and photochemical behavior, Inorg. Chem. Commun. $2011 \quad \mathbf{1 4}, \quad 1756-1761 \quad$ DOI: 10.1016/j.inoche.2011.08.003
[16] Valicsek, Z.; Horváth, O.: Formation, photophysics and photochemistry of thallium(III) 5,10,15,20tetrakis(4-sulphonatophenyl)porphyrin: New supports of typical sitting-atop features, J. Photochem. Photobiol. A, 2007

186, $1-7$

DOI: 